\title{
石笋密度蕴含的过去气候变化信息：以末次冰消期黄土 高原西部武都万象洞石笋为例
}

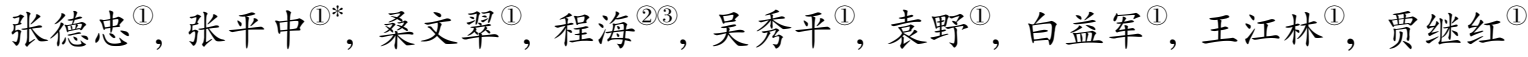 \\ (1) 兰州大学资源环境学院，西部环境教育部重点实验室，兰州 730000; \\ (2) 西安交通大学全球环境变化研究院, 西安 710049; \\ (3) Department of Geology and Geophysics, University of Minnesota, Minneapolis, MN 55455, USA \\ * 联系人, E-mail: pzzhang@1zu.edu.cn
}

2010-05-04 收稿, 2010-09-07 接受

国家自然科学基金(40973007, 40772110)和教育部博士点基金(200807300030)资助项目

摘要 亚洲季风区边缘甘肃武都万象洞一根石笋(WX42A) 在末次冰消期不同生长时期密度变 化表现出有规律的波动。高精度 ${ }^{230} \mathrm{Th}$ 测年和高分辨率的石笋密度结果表明, 生长于 17644 12758 a BP 之间石笋密度变化时间序列在长时间尺度上与反映亚洲季风强度变化的石 笋 $\delta^{18} \mathrm{O}$ 记录变化趋势非常一致, 显示出季风强度增强/减弱 $\left(\delta^{18} \mathrm{O}\right.$ 值偏轻/偏重), 石笋密度随之 增大/减小的特征; 在短时间尺度上石笋密度的降低也对应于一些季风减弱事件如 Inter-Allerød Cold Period (IACP)、Older Dryas (OD) 和 Inter-Bølling Cold Period (IBCP)。总体上, 石笋密度的

\section{关键词}

石笋密度

亚洲季风

气候变化

万象洞

季风边缘区

降低是对季风减弱、降水量减少造成洞穴滴水速率降低、结晶核减少和晶体增大、生物活动强 度减弱和土壤 $\mathrm{CO}_{2}$ 分压降低、碎屑杂质物质增多的反映。然而在季风极端减弱、降水量大幅度 减少和生物活动强度减弱时期, 温度的变化将主导石笋密度的变化, 如在北大西洋 H-1 事件温 度突然降低时, 石笋密度却突然增大, 这是由环境温度的突然降低使得少量下渗水在低温下溶 解的碳酸氢钻浓度升高、溶液过饱和度升高而形成晶体规则紧密排列且密度较大的石笋矿物所 造成的. 这种灵敏记录石笋生长历史和外界环境变化的过去石笋密度随着时间波动, 说明石笋 密度在过去气候变化研究中具有极大的潜力。

洞穴石笋因气候代用指标丰富、定年准确、生长 环境稳定且在生长后基本无后生变化等优点 ${ }^{[1]}$, 已成 为目前最好的过去气候变化研究档案 ${ }^{[2]}$. 其中石笋方 解石的氧碳同位素 ${ }^{[3 \sim 11]}$ 、纹层厚度 ${ }^{[12]}$ 、微量元素 ${ }^{[13 \sim 15]}$ 、 有机质 ${ }^{[16]}$ 等众多指标可以指示降水量、生态演变甚 至大气环流的全球联系. 在亚洲季风区, 高精度高分 辨率石笋记录成功地对高纬极地冰芯时标进行了校

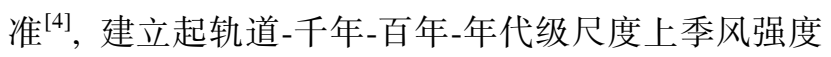
与极地温度、大气温室气体、高纬冰量、海洋环流之 间的桥梁 ${ }^{[3 \sim 11]}$, 而且在亚洲季风变迁与中国朝代更 替、人类活动对亚洲季风的影响 ${ }^{[9]}$ 等方面均取得重要
突破. 由于常用的稳定同位素也常受到洞穴碳酸钙 沉积过程中同位素分馏、降水和温度双重叠加效应等 环境因素的影响 ${ }^{[17,18]}$, 同时岩化作用(如泥晶化作用 和新生变形作用)有时也可以使石笋同位素及微量元 素记录发生偏移 ${ }^{[19]}$. Frisia 等人 ${ }^{[20]}$ 指出在石笋同位素 研究中, 判断石笋记录的气候信号是否经过后生岩 化作用的影响至关重要, 但是目前石笋研究中很少 考虑岩化作用的影响 ${ }^{[19]}$. 因此, 更多石笋气候代用 指标的开发及其相互关系的研究将能够更准确地揭 示气候变化的过程和机制.

Gonzalez 等人 ${ }^{[21]}$ 根据方解石沉积形态学研究表

英文版见: Zhang D Z, Zhang P Z, Sang W C, et al. Implications of stalagmite density for past climate change: An example from stalagmite growth during the last deglaciation from Wanxiang Cave, western Loess Plateau. Chinese Sci Bull, 2010, 55, doi: 10.1007/s11434-010-4190-4 
明, 方解石沉积过程中结晶习性主要受控于水体中 碳酸氢钙过饱和度和雉晶(结晶核)多少. 快速流动 的碳酸氢䥻过饱和水能产生大量的结晶核, 因而能 形成数量众多的等轴晶体, 使得形成结晶致密和密 度较大的石笋; 而当水体流动减弱时, 碳酸氢钙饱 和度过高 (大于六倍饱和度时), 方解石主要以偏三 角体、斜方六面体、六边形柱状体等晶形为主, 而且 容易形成含缺陷晶体较多的枝状结构的方解石晶体 组织, 这将会导致形成的方解石质地疏松和石笋密 度的降低.

北半球气候在 19 10 ka BP 之间经历末次盛冰期 (LGM) 到全新世间冰期 ${ }^{[22]}$ 的转换, 期间又经历了数 次千年-亚轨道尺度上的降温事件如 Heinrich 1 事件 (H1), Younger Dryas 事件 (YD) 和 Bølling-Allerød (B/A)暖事件. 在这些冷暖事件交互发生过程中, 地 表温度 ${ }^{[22]}$ 、降水 ${ }^{[4]}$ 和土壤植被 ${ }^{[23]}$ 等环境因素发生巨大 变化, 这些变化应该造成该时期生长的石笋密度波 动，而且这些密度上的差异应该与降水、温度等气候 变化存在密切联系.

本文以黄土高原西部甘肃武都万象洞内生长在 末次冰消期(17.6 12.8 ka BP)的一根石笋为研究对 象, 在高精度铀系定年基础上, 通过样品计数和对块 状石笋样品称量分别获得高分辨率石笋结晶密度信 息, 讨论洞穴石笋密度变化的影响因素并与亚洲季 风区其他石笋同位素记录对比，尝试解译万象洞石 笋密度变化所蕴含的过去气候变化信息, 并分析石 笋密度作为一个新气候代用指标的可行性.

\section{1 样品与实验}

\section{1 洞穴概况}

甘肃武都万象洞 $\left(33^{\circ} 19^{\prime} \mathrm{N}, 105^{\circ} 00^{\prime} \mathrm{E}\right.$, 海拔 $\left.1200 \mathrm{~m}\right)$ 发育在石炭纪灰岩中, 洞顶上覆土壤主要以黄土为 主，植被发育状况良好. 目前开发的洞穴长约 1100 $\mathrm{m}$, 洞内次生碳酸盐非常发育, 且有大量的现代沉 积 ${ }^{[11,24]}$. 放置在洞穴内部的温度湿度连续监测仪显 示, 洞穴内温度基本恒定在 $11^{\circ} \mathrm{C}(2001$ 年 8 月至 2003 年 1 月), 相对湿度达 $100 \%{ }^{[11,24]}$. 而万象洞所在 地武都年均温(MAT) $12.6^{\circ} \mathrm{C}$, 降水多集中在 5 9 月, 年均降水量 $(\mathrm{MAP}) 489 \mathrm{~mm}$, 属于典型的季风性半干 旱气候(MAT 和 MAP 为 1944 年至 1990 年平均, 数 据来自 Global Historical Climatological Network Version 2, http://www.ncdc.noaa.gov/oa/climate/ghen-mo- nthly/index.php).

\section{2 样品剖面沉积特征}

所研究的石笋样品 WX42A(图 1)于 2003 年 6 月 采自万象洞内距离洞口约 $1000 \mathrm{~m}$ 处. 样品全长 $365 \mathrm{~mm}$, 整体上石笋剖面颜色呈淡黄色，结晶致密，没有明显 的后生变化, 生长纹层清晰可见、平直, 有利于平行 样品纹层高分辨率采样. 自顶部向下至 $30 \mathrm{~mm}$, 颜色 由黄色逐渐变浅, 其中 $25 \mathrm{~mm}$ 处有一明显的浅黄色 条纹, 另外在 $45 \mathrm{~mm}$ 处又有一明显的乳白色条纹, 经 ${ }^{230} \mathrm{Th}$ 定年证明为两个明显的沉积间断. 样品自 $45 \mathrm{~mm}$ 至 $110 \mathrm{~mm}$ 颜色逐渐加深, 其中 150 $230 \mathrm{~mm}$ 之间样 品孔隙比较发育, 清晰可见的纹层至 $330 \mathrm{~mm}$ 处结束; 在石笋顶部与 $18.5 \mathrm{~mm}$ 之间, 可能存在几个沉积间 断, 所以本文选取 18.5 330 $\mathrm{mm}$ 之间的石笋样品作 为主要研究对象.

\section{3 室内样品精细采样方法}

利用牙钻与刻刀相结合的方法在室内进行精细 样品采集 ${ }^{[25]}$. 首先, 在石笋的剖面上生长轴附近用 钢锥自顶至底刻划出两条间距 $10 \mathrm{~mm}$ 且垂直于生长 纹层的直线，然后用牙钻沿这两条画好的直线垂直 石笋剖面向下钻 $5 \mathrm{~mm}$, 形成两个平行的细槽. 在石 笋的顶面上，同样用牙钻垂直生长面向下钻 1 2 mm

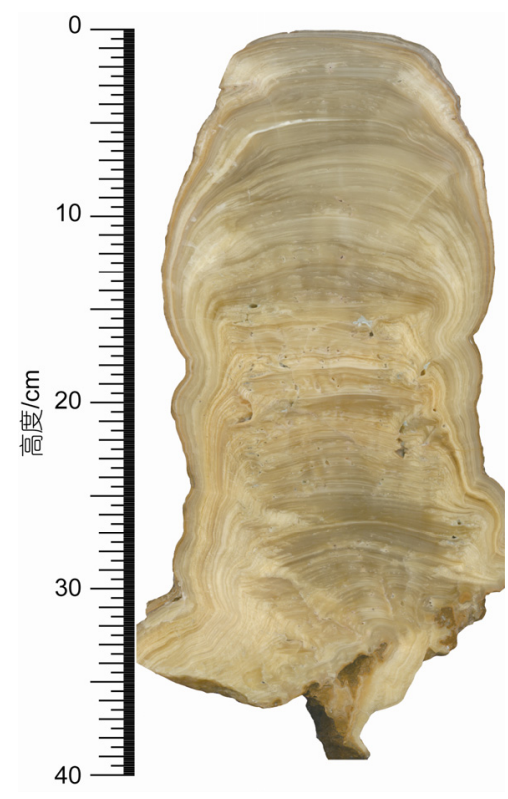

图 1 万象洞石笋 WX42A 剖面图 
深的槽, 并保证这条刻槽平行于石笋剖面. 这样, 石 笋顶面上就形成了由这 3 条刻槽与剖面和顶面的交 线形成的 $10 \mathrm{~mm} \times 5 \mathrm{~mm}$ 的矩形区域, 即为采样面. 用牙钻在样品采集前刻出采样面的作用是用来保证 整个采样过程中采样面的大小不发生改变, 另外刻 槽工作应在采样工作中从上到下逐步进行, 避免一 次刻槽过长引起采样面内石笋样品断裂脱落. 用刻 刀在采样面上平行生长纹层垂直于生长轴自顶部开 始精细刮取样品, 刮取的每个样品重量约为 $30 \mathrm{mg}$, 而且每个样品之间重量差保持在 $\pm 1 \mathrm{mg}$ 之内, 这样每 毫米所采集的样品个数就可以代表石笋的沉积密度, 并将这个样品数称为密度指数 $($ 个 $/ \mathrm{mm})$. 在 $\mathrm{WX} 42 \mathrm{~A}$ 中共刮取 7857 个小样品, 按照密度指数的定义, 本 样品共获得密度指数数据 306 个, 实验误差主要由单 个样品的质量差和采样过程中的损失以及采样面大 小的细微变化所造成, 整体误差不超过 5\%.

同时, 我们自石笋顶部 $18.5 \mathrm{~mm}$ 起沿生长轴每 5 毫米(底部 250 310 mm 部分由于受样品限制每 10 毫 米)采取一个块状样品, 共计 53 个, 借助一个 $25 \mathrm{~mL}$ $\left(V_{\mathrm{s}}\right)$ 容量瓶和电子天平计算块状样品的密度. 首先, 对待测样品用电子天平称量以获得其重量 $\left(m_{\mathrm{s}}\right)$, 然后 在 $25 \mathrm{~mL}$ 容量瓶中注人蒸馏水、定容, 并用电子天平 称量得出容量瓶和水的重量 $\left(m_{\mathrm{w}}\right)$; 再将块状样品放 人盛有水的容量瓶内定容并称得总重量 $\left(m_{\mathrm{sw}}\right)$; 最后 将样品取出并将水倒空后再称空容量瓶的重量 $\left(m_{\mathrm{p}}\right)$. 这样, 小块状石笋样品的密度就可以通过下面公式 求出

样品密度: $D_{\mathrm{s}}=m_{\mathrm{s}} /\left\{\left[\left(m_{\mathrm{s}}+m_{\mathrm{w}}-m_{\mathrm{sw}}\right)\right] /\left[\left(m_{\mathrm{w}}-m_{\mathrm{p}}\right) / V \mathrm{~s}\right]\right\}$, 单位为 $\mathrm{g} / \mathrm{cm}^{3}$.
实验误差主要由称量过程中电子天平的称量误 差以及定容误差所造成, 样品称量误差 $\leqslant 0.1 \%$, 定容 误差 $\leqslant 1 \%$ ，整体误差应小于 $3 \%$.

\section{4 定年方法}

$\mathrm{U}$ 系测年样品用 $0.5 \mathrm{~mm}$ 牙钻沿石笋生长纹层钻 取, 在美国明尼苏达大学地质与地球物理系地质年 代学实验室测定, 测试方法见文献[26,27], 分析仪器 为 MC-ICP-MS, 年龄误差为 $\pm 2 \sigma$.

\section{2 结果与讨论}

\section{$2.1 \mathrm{WX42 \textrm {A }}$ 的 ${ }^{230} \mathrm{Th}$ 年代模式}

石笋 WX42A 在 $18.5 \sim 330 \mathrm{~mm}$ 之间的 10 个 ${ }^{230} \mathrm{Th}$ 年代数据见表 1. 所测定年代的样品 $U$ 含量非常高, 在 $2.6 \times 10^{-6} \sim 5.1 \times 10^{-6} \mathrm{~g} \cdot \mathrm{g}^{-1}$ 之间(平均为 $3.2 \times 10^{-6} \mathrm{~g} \cdot \mathrm{g}^{-1}$ ), 所以获得了精确的年代数据(误差在 50 100 a 之间, 平均误差为 66 a). 年代结果表明, WX42A 样品生长 时段处于 17644 12758 a BP 的末次冰消期. 我们采 用线性内插法建立了该石笋研究层段内的年代模型 (图 2). WX42A 石笋在 $331.5 \mathrm{~mm}$ (17644 a BP) 48.5 $\mathrm{mm}$ (13904 a BP)之间沉积速率的变化幅度较小 (平均 沉积速率约为 $0.076 \mathrm{~mm} / \mathrm{a}$ ), 可是分别在 $247.5 \mathrm{~mm}$ (16484 a BP) 203.5 mm (15996 a BP)和 $203.5 \mathrm{~mm}$ (15996 a BP) 181 mm (15659 a BP)之间出现了沉积 速率的增加和降低. 在 $48.5 \mathrm{~mm}$ (13904 a BP) 18.5 mm (12758 a BP)之间, 沉积速率大幅度降低, 并且 在 47 和 $26.5 \mathrm{~mm}$ 处分别出现了两个持续时间约为 80 和 $220 \mathrm{a}$ 的沉积间隙, 可能与洞穴滴水的减少有关.

表 1 万象洞石笋 WX42A 的 MC-ICP-MS 铀系测年结果 ${ }^{\text {a) }}$

\begin{tabular}{|c|c|c|c|c|c|c|c|}
\hline 样品编号 & 距离/mm & $\begin{array}{c}{ }^{238} \mathrm{U} \\
\left(\times 10^{-9}\right) \\
\end{array}$ & $\begin{array}{c}{ }^{232} \mathrm{Th} \\
\left(\times 10^{-12}\right)\end{array}$ & $\begin{array}{c}\delta^{234} \mathrm{U} \\
\text { 测量值 }\end{array}$ & $\begin{array}{c}{ }^{230} \mathrm{Th} /{ }^{238} \mathrm{U} \\
\text { 活度比 }\end{array}$ & $\begin{array}{c}230 \mathrm{Th} \text { 年龄/a } \\
\text { 未校正 }\end{array}$ & $\begin{array}{c}{ }^{230} \mathrm{Th} \text { 年龄/a BP } \\
\text { 校正年龄 }\end{array}$ \\
\hline WX42A-1 & 18.5 & $3574 \pm 6$ & $1802 \pm 46$ & $1132 \pm 2$ & $0.2384 \pm 0.0009$ & $12819 \pm 50$ & $12758 \pm 50$ \\
\hline WX42A-2 & 26.2 & $2535 \pm 9$ & $20990 \pm 93$ & $1176 \pm 5$ & $0.2538 \pm 0.0014$ & $13400 \pm 90$ & $13236 \pm 100$ \\
\hline WX42A-3 & 26.8 & $3072 \pm 5$ & $855 \pm 50$ & $1184 \pm 2$ & $0.2557 \pm 0.0010$ & $13450 \pm 60$ & $13396 \pm 60$ \\
\hline WX42A-4 & 46.5 & $2567 \pm 5$ & $1413 \pm 64$ & $1205 \pm 3$ & $0.2648 \pm 0.0012$ & $13810 \pm 70$ & $13749 \pm 70$ \\
\hline WX42A-5 & 48.5 & $2809 \pm 5$ & $9877 \pm 56$ & $1200 \pm 2$ & $0.2676 \pm 0.0010$ & $14004 \pm 56$ & $13904 \pm 61$ \\
\hline WX42A-6 & 145 & $5059 \pm 9$ & $5508 \pm 48$ & $1237 \pm 2$ & $0.2954 \pm 0.0010$ & $15271 \pm 55$ & $15203 \pm 56$ \\
\hline WX42A-7 & 181 & $2746 \pm 5$ & $2093 \pm 39$ & $1199 \pm 3$ & $0.2985 \pm 0.0011$ & $15727 \pm 65$ & $15659 \pm 66$ \\
\hline WX42A-8 & 203.5 & $2784 \pm 5$ & $1115 \pm 35$ & $1177 \pm 3$ & $0.3013 \pm 0.0010$ & $16055 \pm 63$ & $15996 \pm 63$ \\
\hline WX42A-9 & 247.5 & $3450 \pm 6$ & $2862 \pm 36$ & $1210 \pm 3$ & $0.3147 \pm 0.0011$ & $16550 \pm 63$ & $16484 \pm 64$ \\
\hline WX42A-10 & 331.5 & $3369 \pm 6$ & $6121 \pm 33$ & $1174 \pm 3$ & $0.33 \pm 0.0010$ & $17722 \pm 64$ & $17644 \pm 65$ \\
\hline
\end{tabular}

a) $\lambda_{230}=9.1577 \times 10^{-6} \mathrm{a}^{-1}, \lambda_{234}=2.8263 \times 10^{-6} \mathrm{a}^{-1}, \lambda_{238}=1.55125 \times 10^{-10} \mathrm{a}^{-1} . \delta^{234} \mathrm{U}=\left(\left[\left[^{234} \mathrm{U} / /^{238} \mathrm{U}\right]\right.\right.$ 活度比 -1$) \times 1000 . \delta^{234} \mathrm{U}$ 初姑值根据 ${ }^{230} \mathrm{Th}$ 年龄 $(T)$

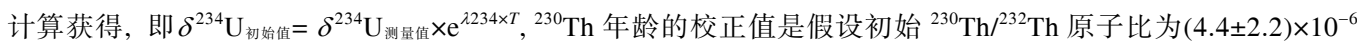




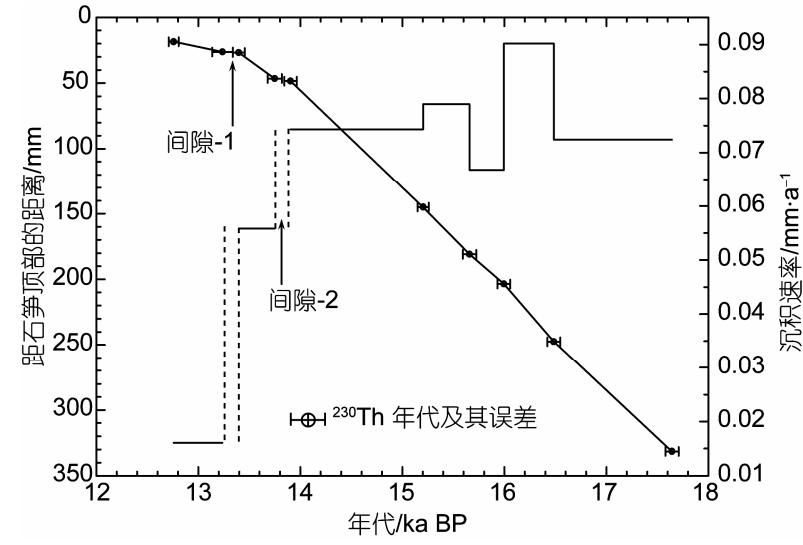

图 2 万象洞石笋 WX42A 的年代模式和沉积速率分布图 虚线指示了间隙- 1 和间隙- 2 的持续时间

\subsection{WX42A 的密度结果}

图 3 呈现了两种方法所描述的石笋样品高分辨 率密度测试结果随时间的变化序列(密度指数和块状 密度的分辨率分别是 16 和 $87 \mathrm{a}$ ). 密度指数曲线与块 状密度曲线两者基本上都显示出一致的变化趋势, 并以 14800 a BP 为界分为 2 阶段( I 和 II ), 表明我们 通过两种不同方法获得的密度曲线可以真实准确地 反映石笋密度的变化状况. 在 17644 14800 a BP 期
间的第 II 阶段，密度指数和块状密度都显示了低值， 变化范围分别是 10 34 个 $/ \mathrm{mm}$ (平均值为 20 个 $/ \mathrm{mm}$ )和 $2.4 \sim 3.7 \mathrm{~g} / \mathrm{cm}^{3}$ (平均值为 $2.8 \mathrm{~g} / \mathrm{cm}^{3}$ ); 而在 $14800 \sim$ 12758 a BP 期间( I 阶段), 它们分别在 20 44 个 $/ \mathrm{mm}$ (平均值为 32 个 $/ \mathrm{mm}$ ) 和 $2.6 \sim 4.7 \mathrm{~g} / \mathrm{cm}^{3}$ (平均值为 3.3 $\left.\mathrm{g} / \mathrm{cm}^{3}\right)$ 之间变化, 且密度平均值较高.

密度指数曲线自 LGM 以来, WX42A 在整个生长 时段内清晰的显示出 7 个明显的密度增加峰值. 它们 的时代主要集中在 $17070,16540,15940,15420,14500$, 14010 和 12940 a BP 左右; 同样地, 块状密度曲线也 显示 7 个明显的峰值, 但是它们峰值的中心年代与密 度指数曲线略有差异, 可能由于块状密度分辨率较 低，每个数据的跨时较大所造成.

\section{3 石笋密度的气候与环境含义}

如上所述，水体流动性和碳酸氢钻溶液的过饱 和度是石笋密度变化的主要因素. 可是, 它们直接受 多种环境因素的影响, 如降水量、温度、洞穴上覆土 壤类型及植被的变化等都会造成洞穴滴水的过饱和 度和结晶核多少的变化，而洞穴上覆土层及植被以 及下渗水通道的变化则会影响水体中杂质物质及微 量元素的含量从而可改变石笋形成过程中结晶状况

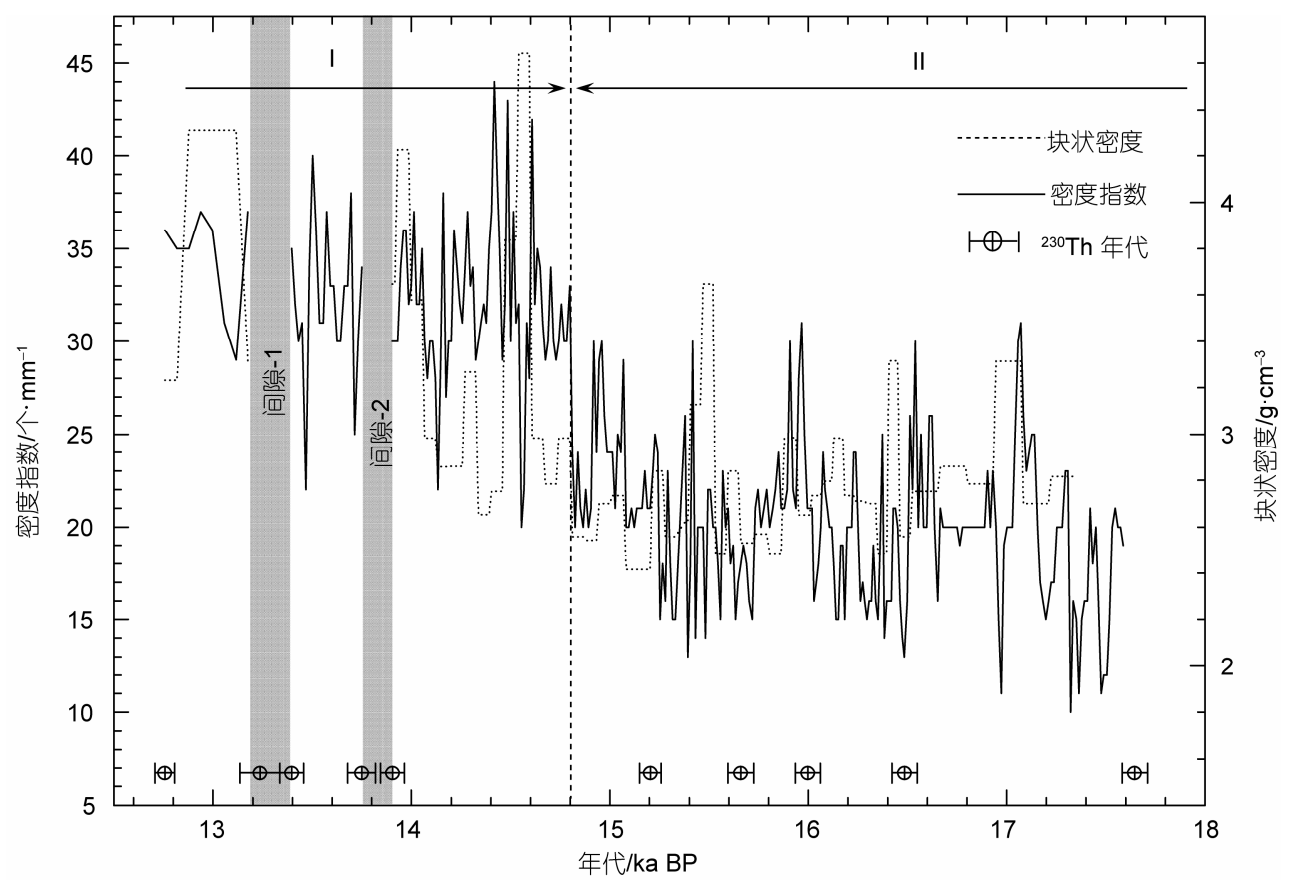

图 3 万象洞石笋 $\mathrm{WX42A}$ 的密度记录和 ${ }^{230} \mathrm{Th}$ 年龄及误差范围

图中两条阴影标示了 WX42A 顶部的两个沉积间断 
和包裹体的形成, 进而影响石笋的密度 ${ }^{[13,21,28 ~ 34]}$. 因 此, 过去石笋形成环境的变化将会导致石笋密度随 过去时间的变化.

\section{1 环境温度对石笋密度的影响}

洞穴温度一般与当地的年平均温度密切相关 ${ }^{[1]}$. 碳酸钙在酸性-弱酸性水中的溶解度随温度升高而降 低, 所以当外界温度发生快速变化时, 将会造成由于 洞穴滴水碳酸氢钙过饱和度不同而发生沉积状况的 改变. 石笋生长模拟实验表明 ${ }^{[29]}$, 碳酸氢钙溶液温 度越低, 基底温度越高, 溶液过饱和度将会越高, 碳 酸钙的生长速率就越快, 越容易形成规则的斜方六 面体且排列紧密的晶体, 使得石笋的密度增大.

\section{2 微量元素对石笋密度的影响}

在石笋沉积过程中, 洞穴滴水的化学性质以及 大气二氧化碳分压变化也会导致洞穴沉积物中微量 元素的含量发生变化 ${ }^{[13,30,31]}$. 例如, 锌含量与钻含量 呈负相关关系, 方解石中锌含量在石笋亮色区域中 低而在暗色区域中高 ${ }^{[32]}$, 同时锌含量增加会导致石 笋变得更粗粘、疏松并含有更多孔隙 ${ }^{[33]}$. 实验表明, 锌含量随水溶液中氯离子的增加而增加, 在晶格中 的排列与大离子 $\mathrm{Sr}^{2+}$ 和 $\mathrm{Ba}^{2+}$ 模式相同 ${ }^{[32]}$. 因此, 降水 减少时, 洞穴上覆土壤中水含量减少, 盐分得不到足 够的稀释, 使得洞穴滴水中氯离子含量增加, 洞穴沉 积物中锌离子含量也随之增加, 造成石笋密度的降 低. 同时, 在石笋样品颜色较深部分的铁含量增加也 可明显导致石笋密度的降低 ${ }^{[34]}$. 总之, 降水减少, 微 量元素含量增加, 导致石笋密度的降低.

\section{3 杂质及包裹体对石笋密度的影响}

通过显微镜对以色列 Soreg 洞石笋岩石学特征 全面分析以及与同位素系列的对比发现 ${ }^{[28,35]}$, 末次 冰期与随后间冰期的石笋沉积结构有显著差别. 石 笋 $\delta^{18} \mathrm{O}$ 相对稳定阶段出现在由平行消光并具有优势 方位 (垂直生长微层理)的方解石晶体组成的石笋浅 色纹层部分, 且碎屑物质含量 $<0.1 \%$; 当 $\delta^{18} \mathrm{O}$ 出现短 时间尺度波动时, 石笋颜色加深, 碎屑物质增加, 以 随机分布的等轴的小晶体为主, 碎屑物质及氧化物 含量高达 $0.5 \%$, 使得石笋透明度显著下降, 杂质及 包裹体含量增加, 石笋密度降低.

\section{4 洞穴上覆植被和土壤变化对石笋密度的影响}

温度升高, 降水量增大, 洞穴上覆植被和土壤发 育将得到改善 ${ }^{[36,37]}$. 这样大气降水在洞穴上覆土壤 层中的滞留时间增加, 生物活动的加强, 土壤 $\mathrm{CO}_{2}$ 分 压增加, 土壤水的 $\mathrm{pH}$ 降低 ${ }^{[37 \sim 40]}$, 使得土壤水溶解碳 酸钻的能力大大加强, 为洞穴内次生碳酸盐的形成 提供了过饱和度较高、且携带丰富凝结核的下渗水 源, 形成晶体排列规则、晶体较小、杂质和空隙较少 的密度较大的石笋. 反之亦然.

总之, 石笋密度的差异与洞穴外部环境因素密 切相关, 通过石笋密度的研究我可以提取与石笋生 长过程有关的过去气候变化的信息.

\section{WX42A 密度时间序列与亚洲季风强度 变化的关系}

为了进一步验证万象洞石笋密度与环境因素的 关系, 我们把 WX42A 密度时间序列与反映亚洲季风 强度变化的葫芦洞/董哥洞石笋 $\delta^{18} \mathrm{O}$ 记录 ${ }^{[4,5,8]}$ 进行了 比较(图 4). 发现 WX42A 密度时间序列在长时间尺 度上与季风强度的变化趋势非常一致, 说明万象洞 石笋密度的差异受控于亚洲季风强度的变化. 在末 次冰消期内, 17644 12758 a BP, 石笋 WX42A 密度逐 渐增大, 这种趋势与北半球夏季太阳辐射强度 ${ }^{[41]}$ 变 化一致, 也与反映热带辐合带(ITCZ)位置变化的委 内瑞拉北部 Cariaco Basin 反照率记录相似 ${ }^{[42]}$, 支持 亚洲季风受控于热带辐合带(ITCZ)位置变化的观 点 ${ }^{[5,7,8]}$. 特别在 $B / A$ 转换时, 石笋密度的逐渐增大紧 密地匹配于季风强度的逐渐加强(图 4). 可是, 在以 14800 a BP 为界的 WX42A 密度时间序列 2 个明显变 化阶段中(图 3 和 4), 石笋密度与葫芦洞石笋 $\delta^{18} \mathrm{O}$ 记 录在峰谷关系上表现出不同的对应关系, 14800 a BP 之后的第 I 阶段中表现为相反的负相关关系, 即石 笋密度的增大对应于偏负的葫芦洞石笋 $\delta^{18} \mathrm{O}$ 值, 而 在 14800 a BP 前的第 II 阶段中则表现为石笋密度的 峰值对应于偏正的葫芦洞石笋 $\delta^{18} \mathrm{O}$ 值. 通过计算万 象洞石笋密度指数与葫芦洞 $\mathrm{H} 82$ 石笋 $\delta^{18} \mathrm{O}$ 的相关系 数, 也显示石笋密度在 14800 12758 a BP 期间( I )内 在短时间尺度上与葫芦洞石笋 $\delta^{18} \mathrm{O}$ 呈现出负相关关 系 $(r=0.214, n=98, P=0.05)$, 而在 17644 14800 a BP 期间 (II), 总体上相对低密度的石笋沉积却显示出正 相关关系 $(r=0.264, n=208, P=0.01)$, 与 2 条曲线 


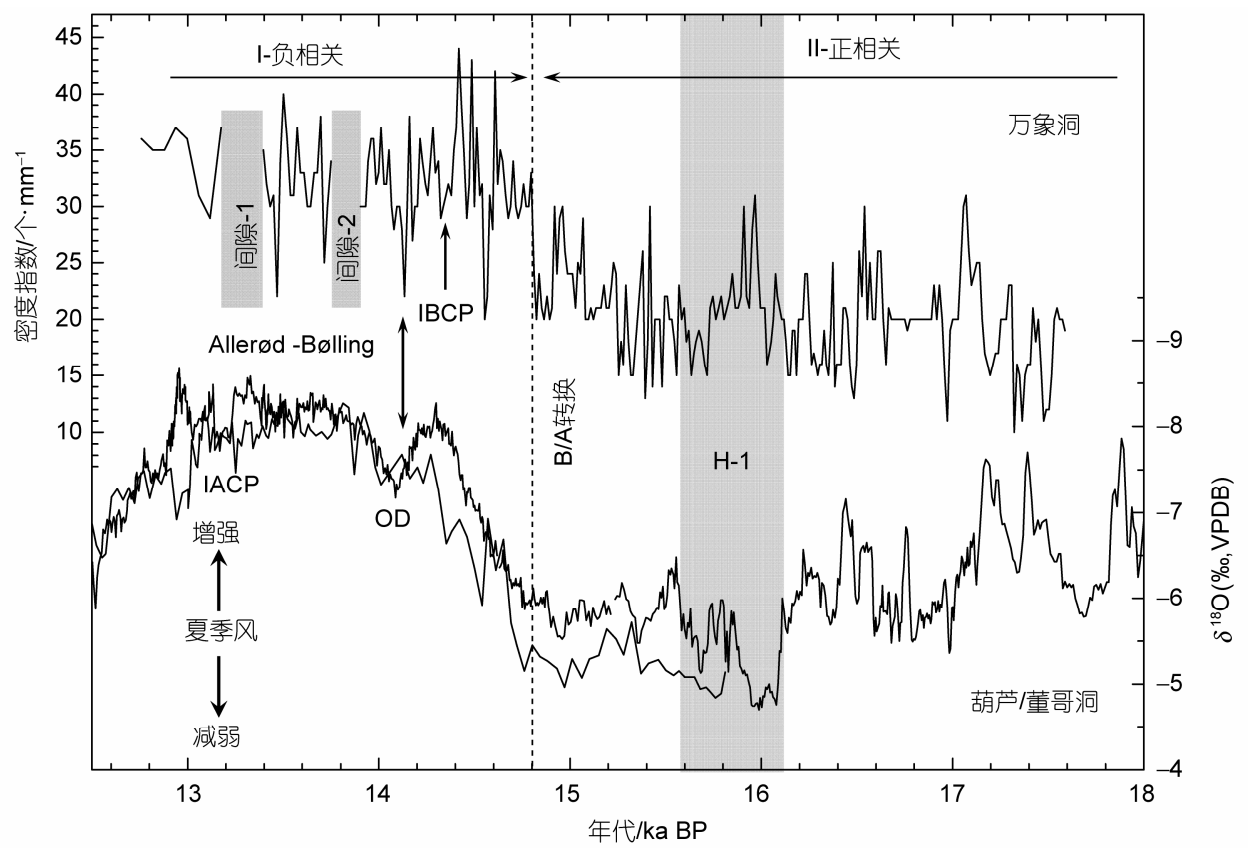

图 4 万象洞石笋 WX42A 密度记录与葫芦/董哥洞石笋 $\delta^{18} O$ 记录的对比

上下两条曲线分别为万象洞石笋 $\mathrm{WX} 42 \mathrm{~A}$ 密度指数记录和葫芦洞石笋 $\mathrm{H} 82$ (长记录)以及董哥洞石笋 $\mathrm{D} 4$ (短记录) $\delta^{18} \mathrm{O}$ 记录 ${ }^{[8]}$; 垂直虚线标出了万 象洞石笋密度指数与葫芦洞石笋 $\delta^{18} \mathrm{O}^{[4,5]}$ 记录正/负相关的界限, 较长的阴影区标出了 $\mathrm{H}-1$ 冷事件的持续时间, 两条短阴影标示了 WX42A 顶部 的两个沉积间断

峰谷对应关系相一致.

阶段 II (17644 14800 a BP), 石笋密度偏低(图 3 和 4), 总体上与偏正的石笋 $\delta^{18} \mathrm{O}$ 时期相对应(图 4), 并在 16284 a BP $(230 \mathrm{~mm}) \sim 15266$ a BP $(150 \mathrm{~mm})$ 期间 沉积了孔隙比较发育的石笋(图 1), 处于季风减弱期. 这是由季风减弱和降水量减少造成洞穴滴水速率降 低、结晶核减少和晶体增大、生物活动强度减弱和土 壤 $\mathrm{CO}_{2}$ 分压降低、碎屑杂质物质增多，导致石笋密度 降低的结果 ${ }^{[21,28,37 ~ 39]}$. 可是这一时期密度指数在短时 间尺度上与葫芦洞石笋 $\delta^{18} \mathrm{O}$ 记录 ${ }^{[4,5]}$ 正相关, 即增加 的密度对应于偏重的 $\delta{ }^{18} \mathrm{O}$ 值, 与总体趋势相反. 这 种关系可以用温度的突然降低或升高, 造成石笋密 度的增大或减小解释. 在这个时期, 高纬北大西洋地 区温度变化超过了太阳辐射所驱动的季风强度变化 的影响 ${ }^{[43]}$, 温度主宰着这个时期万象洞石笋密度的 变化, 而且出现突发性降温事件(H-1). 因此, 环境温 度的突然降低, 使得少量下渗水在低温下溶解的碳 酸氢钙浓度升高, 由于万象洞良好的封闭性导致洞 穴温度在短时间内仍然处于恒温状态, 在环境温度 显著降低时洞穴内温度将相对保持较高温度, 从而 使得溶液过饱和度升高, 有利于形成晶体规则紧密
排列且密度较大的石笋矿物 ${ }^{[21]}$. 其中最典型的是大 约发生在 16100 15570 a BP 之间(持续约 530 a) 的 WX42A 石笋样品孔隙明显减少, 颜色变深且质地更加 致密, 密度指数显著增大, 与葫芦洞石笋 $\delta^{18} \mathrm{O}$ 记录 正相关, 是北大西洋 H-1 剧烈降温事件的气候信号跨 越西风带沿近地表快速传播到亚洲季风区 ${ }^{[44]}$ ，引起 万象洞地区的快速降温, 从而造成石笋密度的增大.

阶段 I (14480 12758 a BP)，石笋整体密度明显 大于阶段 II (图 3 和 4), 可能是对石笋密度控制因素 发生重大变化的反映.

一般情况下, 快速流动的饱和碳酸盐水能够形 成等轴随机分布的方解石晶体，由于流动较快的水 能够携带更多的土壤黏土颗粒和碎屑氧化物，容易 产生大量的结晶核; 相反地, 在低滴水速率的条件 下，结晶核较少，晶体能够得到较好的生长空间，发 育完全, 同时水流缓慢, 携带的土壤微粒较弱, 因此 石笋中的黏土物质含量较低. 即水动力较小时, 由厚 的、粗粒晶体、浅色的和具有优势方位的方解石组成, 而且具优势方位的大晶体被微米级晶体组成的薄层 所分隔 ${ }^{[28]}$.

该时期洞穴滴水流动性增加，碳酸钙结晶核增 
多, 单个方解石晶体变小, 且晶形规则 ${ }^{[21]}$, 可能是造 成石笋密度增大的原因. 而且, 这一时期明显的石笋 密度变化与葫芦洞石笋 $\delta^{18} \mathrm{O}$ 记录的负相关关系(图 4), 说明北半球气候进人 $\mathrm{B} / \mathrm{A}$ 暖期后, 亚洲季风增强、温 度升高、降水量增大、地表生物活动增强, 有利于高 密度石笋的生长. 同时, 这一时段内, WX42A 石笋密 度指数也表现出更为强烈的短时间尺度上的波动, 出现了持续时间较短并集中在 $14550 ， 14320 ， 14130$, $13710,13460,13120$ a BP 左右的 6 个密度较小的时 期, 其中集中在 14320,14130 和 13120 a BP 左右的 3 个密度低值期分别对应于格陵兰 GISP2 冰芯 $\delta^{18} \mathrm{O}$ 记 录 ${ }^{[22]}$ 中的 IBCP, OD 和 IACP. 除了 IBCP 以外, OD 和 $\mathrm{IACP}$ 在葫芦洞 ${ }^{[4,5]}$ 、董哥洞 ${ }^{[8]}$ 石笋记录中均能找到 $\delta^{18} \mathrm{O}$ 变重的时期, 说明石笋密度的差异在短时间尺度上 也灵敏的记录亚洲季风强度的变化.

\section{5 结论}

在 17644 12758 a BP 之间生长于季风边缘区黄 土高原西部的万象洞石笋密度的变化详细地记录了 末次冰消期亚洲季风强度的变迁. 在长时间尺度上
它与反映亚洲季风强度变化的石笋 $\delta^{18} \mathrm{O}$ 记录的变化 趋势非常一致, 显示出季风强度增强/减弱 $\left(\delta^{18} \mathrm{O}\right.$ 值 偏轻/偏重), 石笋密度随之增大/减小的特征, 与降 水量、温度、地表生物活动强度密切相关; 同时短 时间尺度上石笋密度的降低和一些季风减弱事件也 有良好的相关性, 如 IACP, OD 和 IBCP, 是季风减 弱、降水量减少造成洞穴滴水速率降低、结晶核减 少和晶体增大、生物活动强度减弱和土壤 $\mathrm{CO}_{2}$ 分压 降低、碎屑杂质物质增多的反映. 而且当环境温度 发生突然变化时, 它将主导石笋密度的变化, 这种 情况主要发生在季风极端减弱、降水量大幅度减少 和生物活动强度减弱时期, 如在北大西洋 H-1 事件 发生时, 环境温度突然降低, 石笋密度却突然增大, 这是由温度的突然降低并使得少量下渗水在低温 下溶解的碳酸氢钙浓度升高、溶液过饱和度升高而 形成晶体规则紧密排列且密度较大的石笋矿物所 造成的.

因此，过去石笋密度随着时间的波动，灵敏的记 录了石笋生长的历史和外界环境的变化, 可以成为 过去气候变化研究的有潜力的替代指标.

\section{参考文献}

1 Wigley T M L, Brown M C. The physics of caves. In: Ford T D, Cullingford C H D, eds. The Science of Speleology. London: Academic Press, 1976. 329-358

2 Henderson G M. Caving in to new chronologies. Science, 2006, 313: 620-622

3 Cheng H, Edwards R L, Broecker W S, et al. Ice age terminations. Science, 2009, 326: 248-252

4 Wang Y J, Cheng H, Edwards R L, et al. A high-resolution absolute-dated late Pleistocene monsoon record from Hulu Cave, China. Science, 2001, 294: 2345-2348

5 吴江漟, 汪永进, 程海, 等. 葫芦洞石笋记录的 19.9 17.1 ka BP 东亚夏季风增强事件. 中国科学 D 辑: 地球科学, 2009, 39: 61一69

6 Wang Y J, Cheng H, Edwards R L, et al. The Holocene Asian monsoon: Links to solar changes and north Atlantic climate. Science, 2005, 308: $854-857$

7 Wang Y J, Cheng H, Edwards R L, et al. Millennial- and orbital-scale changes in the east Asian monsoon over the past 224000 years. Nature, 2008, 451: 1090-1093

8 Yuan D X, Cheng H, Edwards R L, et al. Timing, duration, and transitions of the last interglacial Asian monsoon. Science, 2004, 304: 575578

9 Zhang P Z, Cheng H, Edwards R L, et al. A test of climate, Sun, and culture relationships from an 1810-year Chinese cave record. Science, 2008, 322: 940-942

10 姜修洋, 孔兴功, 汪永进, 等. 深海氧同位素 8 期东亚夏季风旋回与事件的湖北神农架洞穴记录. 科学通报, 2010, 55: 698一704

11 刘敬华, 张平中, 程海, 等. 黄土高原西缘在 AD 1875 2003 期间石笋氧同位素记录的季风降水变化与海气系统的联系. 科学通报, 2008, 53: 2801-2808

12 Tan M, Baker A, Genty D, et al. Applications of stalagmite laminae to paleoclimate reconstructions: Comparison with dendrochronology/climatology. Quat Sci Rev, 2006, 25: 2103-2117

13 Huang Y M, Fairchild I J, Borsato A, et al. Seasonal variations in $\mathrm{Sr}, \mathrm{Mg}$ and $\mathrm{P}$ in modern speleothems (Grotta di Ernesto, Italy). Chem Geol, 2001, 175: 429-448

14 Fairchild I J, Treble P C. Trace elements in speleothems as recorders of environmental change. Quat Sci Rev, 2009, 28: 449-468

15 Zhou J Z, Lundstrom C C, Fouke B, et al. Geochemistry of speleothem records from southern Illinois: Development of $\left({ }^{234} \mathrm{U}\right) /\left({ }^{238} \mathrm{U}\right)$ as a 
proxy for paleoprecipitation. Chem Geol, 2005, 221: 1-20

16 McGarry S F, Baker A. Organic acid fluorescence: Application to speleothem palaeoenvironmental reconstruction. Quat Sci Rev, 2000, 19: $1087-1101$

17 Johnson K R, Ingram B L, Warren D S, et al. East Asian summer monsoon variability during marine isotope stage 5 based on speleothem $\delta^{18} \mathrm{O}$ records from Wanxiang Cave, Central China. Palaeogeogr Palaeoclimatol Palaeoecol, 2006, 236: 5-19

18 Dykoski C A, Edwards R L, Cheng H, et al. A high-resolution, absolute-dated Holocene and deglacial Asian monsoon record from Dongge Cave, China. Earth Planet Sci Lett, 2005, 233: 71-86

19 Martín-García R, Alonso-Zarza A M, Martín-Pérez A. Loss of primary texture and geochemical signatures in speleothems due to diagenesis: Evidences from Castañar Cave, Spain. Sediment Geol, 2009, 221: 141-149

20 Frisia S, Borsato A, Fairchild I J, et al. Aragonite-calcite relationships in speleothems (Grotte de Clamouse, France): Environment, fabrics, and carbonate geochemistry. J Sediment Res, 2002, 72: 687-699

21 Gonzalez L A, Carpenter S J, Lohman K C. Inorganic calcite morphology: Roles of fluid chemistry and fluid flow. J Sediment Res, 1992, 62: $382-99$

22 Stuiver M, Grootes P M. GISP2 oxygen isotope ratios. Quat Res, 2000, 53: 277-284

23 Dorale J A, Edwards R L, Ito E, et al. Climate and vegetation history of the Midcontinent from 75 to 25 ka: A speleothem record from Crevice Cave, Missouri, USA. Science, 1998, 282: 1871-1874

24 张平中, 陈一萌, Johnson K R, 等. 甘肃武都万象洞滴水与现代石笋同位素的环境意义. 科学通报, 2004, 49: 1529一 1531

25 杨勋林, 张平中, 陈发虎, 等, 近 $50 \mathrm{a}$ 来青藏高原东部高海拔洞穴现代石笋氧同位素组成及其含义. 科学通报, 2007, 52: 698一 706

26 Shen C C, Edwards R L, Cheng H, et al. Uranium and thorium isotopic and concentration measurements by magnetic sector inductively coupled plasma mass spectrometry. Chem Geol, 2002, 185: 165-178

27 Cheng H, Edwards R L, Hoff J A, et al. The half-lives of uranium-234 and thorium-230. Chem Geol, 2000, 169: 17-33

28 Ayalon A, Bar-Matthews M, Kaufman A. Petrography, strontium, barium, and uranium concentrations, and strontium and uranium isotope ratios in speleothems as palaeoclimatic proxies: Soreq Cave, Israel. Holocene, 1999, 9: 715-722

29 Miyazaki H, Mizutani M, Yamashita T, et al. Growth of calcium carbonate crystal imitating stalagmite growth in nature. Mater Res Bull, 2006, 41: $1272-1278$

30 Frisia S, Borsato A, Fairchild I J, et al. Calcite fabrics, growth mechanisms, and environments of formation in speleothems from the Italian Alps and Southwestern Ireland. J Sediment Res, 2000, 70: 1183-1196

31 Fairchild I J, Borsato A, Tooth A F, et al. Controls on trace element (Sr-Mg) composition of carbonate cave waters: Implications for speleothem climatic records. Chem Geol, 2000, 166: 255-269

32 Reeder R J. Interaction of divalent cobalt, zinc, cadmium, and barium with calcite surface during layer growth. Geochim Cosmochim Acta, 1996, 60: $1543-1552$

33 Temmam M, Paquette J, Vali H. Mn and Zn incorporation into calcite as a function of chloride aqueous concentration. Geochim Cosmochim Acta, 2000, 64: 2417-2430

34 Kuczumow A, Vekemans B, Schalm O, et al. Analysis of speleothems by electron and X-ray microprobes. J Anal At Spectrom, 2001, 16: $90-95$

35 Bar-Matthews M, Ayalon A, Kaufinan A. Late Quaternary palaeoclimate in the Eastern Mediterranean region from stable isotope analysis of speleothems at Soreq Cave, Israel. Quat Res, 1997, 47: 155-168

36 Cerling T E, Quade J, Wang Y, et al. Carbon isotopes in soils and paleosols as ecologic and paleoecologic indicators. Nature, 1989, 341: $138-139$

37 Bond-Lamberty B, Thomson A. Temperature-associated increases in the global soil respiration record. Nature, 2010, 464: 579-582

38 Hou J Z, Tan M, Cheng H, et al. Stable isotope records of plant cover change and monsoon variation in the past 2200 years: Evidence from laminated stalagmites in Beijing, China. Boreas, 2003, 32: 304-313

39 Cosford J, Qing H R, Mattey D, et al. Climatic and local effects on stalagmite $\delta^{13} \mathrm{C}$ values at Lianhua Cave, China. Palaeogeogr Palaeoclimatol Palaeoecol, 2009, 280: 235-244

40 Bar-Matthews M, Ayalon A, Matthews A, et al. Carbon and oxygen isotope study of the active water-carbonate system in a karstic Mediterranean cave: Implications for paleoclimate research in semiarid regions. Geochim Cosmochim Acta, 1996, 60: 337-347

41 Berger A, Loutre M F. Insolation values for the climate of the last 10 million years. Quat Sci Rev, 1991, 10: 297-317

42 Peterson L C, Haug G H, Hughen K A, et al. Rapid changes in the hydrological cycle of the tropical Atlantic during the last glacial. Science, 2000, 290: 1947-1951

43 Zhou H Y, Zhao J X, Feng Y X, et al. Distinct climate change synchronous with Heinrich event one, recorded by stable oxygen and carbon isotopic compositions in stalagmites from China. Quat Res, 2008, 69: 306-315

44 Pauluis O, Czaja A, Korty R. The global atmospheric circulation on moist isentropes. Science, 2008, 321: 1075-1078 